\section{Lamin A/C mutation affecting primarily the right side of the heart}

\author{
Laura Ollila, ' Johanna Kuusisto, ${ }^{2}$ \\ Keijo Peuhkurinen, ${ }^{2}$ Satu Kärkkäinen, ${ }^{2}$ \\ Petri Tuomainen, ${ }^{2}$ Maija Kaartinen, ${ }^{1}$ \\ Olayinka Raheem, ${ }^{3}$ Bjarne Udd, ${ }^{3,4,5}$ \\ Jarkko Magga, ${ }^{6}$ Janne Rapola, ${ }^{1}$ \\ Annukka M. Lahtinen, ${ }^{7}$ Eero Lehtonen, ${ }^{8,9}$ \\ Miia Holmström, ${ }^{10}$ Sari Kivistö, ${ }^{10}$ \\ Elisabeth Widén, ${ }^{11}$ Markku Saksa, ${ }^{12}$ \\ Tiina Heliö ${ }^{1}$
}

${ }^{1}$ Helsinki University Hospital, Department of Cardiology, Helsinki; ' ${ }^{2}$ niversity of Tampere, Tampere; ${ }^{3}$ Neuromuscular Research Unit, Tampere University and University Hospital, Tampere; ${ }^{4}$ Folkhälsan Institute of Genetics and Department of Medical Genetics, Haartman Institute, University of Helsinki, Helsinki; ${ }^{5}$ Neurology Department, Vaasa Central Hospital, Vaasa; 'Oulu University Hospital, Oulu; ${ }^{7}$ Research Programs Unit, Molecular Medicine and Department of Medicine, University of Helsinki, Helsinki; ${ }^{8}$ Department of Pathology, University of Helsinki; ' Laboratory Animal Centre, University of Helsinki, Helsinki; ${ }^{10}$ Department of Radiology, University of Helsinki and HUS Radiology (Medical Imaging Center), Helsinki; ${ }^{11}$ Institute for Molecular Medicine Finland (FIMM), University of Helsinki, Helsinki; ${ }^{12}$ Kanta-Häme Central Hospital, Hämeenlinna, Finland

\section{Abstract}

LMNA mutations are amongst the most important causes of familial dilated cardiomyopathy. The most important cause of arrhythmogenic right ventricular cardiomyopathy (ARVC) is desmosomal pathology. The aim of the study was to elucidate the role of $L M N A$ mutations among Finnish cardiomyopathy patients. We screened 135 unrelated cardiomyopathy patients for $L M N A$ mutations. Because of unusual phenotype, two patients were screened for the known Finnish ARVC-related mutations of desmosomal genes, and their Plakophilin-2b gene was sequenced. Myocardial samples from two patients were examined by immunohistochemical plakoglobin staining and in one case by electron microscopy. We found a new $L M N A$ mutation Phe237Ser in a family of five affected members with a cardiomyopathy affecting primarily the right side of the heart. The phenotype resembles ARVC but does not fulfill the Task Force Criteria. The main clinical manifestations of the mutation were severe tricuspid insufficiency, right ventricular enlargement and failure. Three of the affected patients died of the heart disease, and the two living patients received heart transplants at ages 44 and 47. Electron microscopy showed nuclear blebbing compatible with laminopathy. Immunohistochemical analysis did not suggest desmosomal pathology. No desmosomal mutations were found. The Phe237Ser LMNA mutation causes a phenotype different from traditional cardiolaminopathy. Our findings suggest that cardiomyopathy affecting primarily the right side of the heart is not always caused by desmosomal pathology. Our observations highlight the challenges in classifying cardiomyopathies, as there often is significant overlap between the traditional categories.

\section{Introduction}

Idiopathic dilated cardiomyopathy (DCM) is characterized by left ventricular dilatation and impaired systolic function in the absence of hypertension, coronary artery disease, valvular disease or other apparent cause. It is estimated to be hereditary in $25-50 \%$ of patients. ${ }^{1-3}$ Familial DCM has a diverse genetic background. So far, mutations in more than 40 autosomal genes have been found to cause familial DCM in a typically autosomal dominant inheritance pattern. ${ }^{3,4}$ Genes shown to cause DCM can be grouped by function or cellular location into nuclear envelope proteins, such as Lamin $\mathrm{A} / \mathrm{C}$ (LMNA), emerin and nesprin, cytoskeletal proteins such as dystrophin, and sarcomere genes such as myosin heavy chain and troponin $\mathrm{T}^{3}$ Using the traditional Sanger sequencing a genetic cause for familial DCM patients can be found in about a third of the cases. ${ }^{5}$ The proportion of patients receiving a genetic diagnosis for their disease will likely increase with the use of next generation sequencing techniques as suggested recently by Herman et al. reporting that truncating titin mutations explain approximately $25 \%$ of familial DCM.

Lamin $\mathrm{A} / \mathrm{C}$ has been shown to cause $9 \%$ of DCM leading to heart transplantation in Finland. ${ }^{7}$ Six Lamin A/C mutations leading to heart disease have been previously reported in Finland. ${ }^{78}$ Clinical characteristics most often associated with the Lamin $\mathrm{A} / \mathrm{C}$ mutations that cause DCM are arrhythmias, in particular atrial fibrillation, atrioventricular conduction defects, and bradycardia resulting in a need of a pacemaker, left ventricular dilatation and eventually heart failure. The onset of clinical manifestations of the disease is age-depend-
Correspondence: Laura Ollila and Tiina Heliö, HYKS/Meilahden sairaala, Sydäntutkimusosasto, PL 340, 00029 HUS, Finland.

Fax: +358.9.471.74574.

E-mail: laura.hupa@helsinki.fi; tiina.helio@hus.fi

Key words: $L M N A$, Lamin $\mathrm{A} / \mathrm{C}$, dilated cardiomy opathy, arrhythmogenic right ventricular cardiomyopathy.

Acknowledgements: we would like to thank Sini Weckström for technical assistance.

Contributions: LO, TH, responsible for all aspects of reliability and freedom from bias of presented data and their discussed interpretation; JK, genetic analysis, clinical data acquisition and analysis, manuscript revision; KP, PT, MK, JM, JR, $\mathrm{MH}, \mathrm{SKi}, \mathrm{EW}, \mathrm{MS}$, clinical data acquisition and analysis, manuscript revision; SKä, AL, genetic analysis, manuscript revision; OR, BU, EL, immunohistochemical analysis of endomyocardial samples, manuscript revision.

Funding: Finnish Foundation for Cardiovascular Research, Finnish Medical Foundation, Special governmental subsidy for health sciences research, EVO-funds, Finnish Cultural Foundation.

Conflict of interests: the authors declare no potential conflict of interests.

Received for publication: 5 November 2012.

Revision received: 15 January 2013.

Accepted for publication: 19 January 2013.

This work is licensed under a Creative Commons Attribution NonCommercial 3.0 License (CC BYNC 3.0).

(C) Copyright L. Ollila et al., 2013

Licensee PAGEPress, Italy

Cardiogenetics 2013; 3:e1

doi:10.4081/cardiogenetics.2013.el

ent. Carriers of Lamin A/C mutations have also been shown to be at a high risk of ventricular arrhythmias and sudden death., ${ }^{9,10}$

Arrhythmogenic right ventricular cardiomyopathy (ARVC) affects primarily the right ventricle, although lately biventricular and left ventricular forms have been identified.11,12 ARVC is characterized by life-threatening arrhythmias and the replacement of the myocardium with fibro-fatty tissue. It has been considered to be caused mainly by mutations in genes encoding several desmosomal proteins. ${ }^{13}$ In addition, non-desmosomal genes such as ryanodine receptor 2 (RYR2), transforming growth factor beta 3 (TGFB3), transmembrane protein 43 (TMEM43), and recently LMNA, have been associated with ARVC. ${ }^{14-17}$ Surprisingly, in a population-based cohort of over 6000 Finns, the prevalence of ARVC-related desmosomal mutations was 1:200 suggesting incomplete penetrance. ${ }^{18}$ There is signifi- 
cant overlap between the traditional clinical subtypes of cardiomyopathy. It is not uncommon for a cardiomyopathy patient to have characteristics of more than one clinical subtype, or change phenotype as the disease progresses. The vastly growing genetic knowledge of cardiomyopathies also challenges the rigid division of cardiomyopathies in to the classic categories. ${ }^{1,11}$

In our search for new LMNA mutations in Finnish cardiomyopathy patients we found three new mutations and one that has been previously reported elsewhere. ${ }^{19}$ One of the new mutations is associated with a clinical phenotype affecting primarily the right side of the heart. Another new mutation is located in the same codon as a previously described mutation..$^{20}$

\section{Materials and Methods}

We screened 133 unrelated DCM patients of Finnish origin from the University Hospitals of Helsinki and Kuopio recruited between 1999 and 2007 for $L M N A$ mutations. We also included two unrelated patients who did not fulfil all the criteria for DCM at the time of diagnosis. The available probands carrying Lamin A/C mutations and their adult family members including unrelated spouses were evaluated by physical examination, 12-lead electrocardiograph, appropriate laboratory tests, transthoracic echocardiography (M-mode, two-dimensional and Doppler) and spiroergometry. All the available hospital records of the probands and their family members were acquired.

The genetic analyses concerning Lamin A/C were performed in the Diabetes and Heart Disease Research Unit at the University of Eastern Finland. DNA was extracted from peripheral blood leukocytes and amplified using polymerase chain reaction (PCR). The coding regions of the $L M N A$ gene were directly sequenced with ABIPRISM 310 or 3100 Genetic analyzer (PE Applied Biosystems, Foster City, CA, USA). The pathogenicity of Lamin $\mathrm{A} / \mathrm{C}$ mutations was assessed in silico using Poly Phen-2 software. ${ }^{21}$

The genetic analyses of desmosomal proteins were performed at the University of Helsinki. All protein-coding exons and exonintron junctions of plakophilin-2b (PKP2b) were amplified using PCR and sequenced with BigDye Terminator v3.1 and ABI 3730xl DNA Analyzer (Applied Biosystems, Carlsbad, CA, USA). All five known Finnish ARVC-associated mutations plakophilin-2 (PKP2) Q59L, PKP2 Q62K, PKP2 N613K, desmoplakin (DSP) T1373A, and desmoglein-2 (DSG2) 3059_3062delAGAG were assayed using direct sequencing or restriction enzyme assays as described previously. ${ }^{18,22}$

Myocardial samples of the patients III:4 and III:6 carrying the LMNA Phe237Ser mutation were examined by immunohistochemical analysis for the desmosomal protein plakoglobin. Sections of the myocardial biopsies were stained with plakoglobin antibody (SigmaAldrich, St. Louis, MO, USA) in dilution 1:10,000 using the BenchMark immuno-stainer (Ventana Medical Systems Inc., Tucson, AZ, USA). The stainings were done using the official protocol of the BenchMark immuno-stainer and visualized with a peroxidase based detection kit (UltraView Universal DAB detection kit, Ventana Medical Systems Inc., Tucson, AZ, USA). For electron microscopy, endomyocardial biopsy material was fixed in $2.5 \%$ glutaraldehyde in $0.1 \mathrm{M}$ phosphate buffer, $\mathrm{pH} 7.4$, at room temperature for $2 \mathrm{~h}$. The samples were postfixed in $1 \% \mathrm{OsO}_{4}$ for $1 \mathrm{~h}$, dehydrated in ethanol and embedded in LX112. Semithin sections were stained with toluidine blue. Thin sections were stained with uranyl acetate and lead citrate and examined in a Jeol 1400 electron microscope equipped with Olympus-SIS Morada digital camera.

Parametric linkage analysis was carried out in Pedigree 1 by utilizing the software Merlin..$^{23}$ An affected-only model was used, setting the disease and marker allele frequencies to $1 / 10,000$, and considering family-members with atrioventricular blocks, arrhythmias or DCM as affected. All participants of the study signed written informed consent. The study protocol was approved by the Ethics Committees of the Universities of Helsinki and Eastern Finland, and is in compliance with the Helsinki declaration.

\section{Results}

We found one previously reported Lamin $\mathrm{A} / \mathrm{C}$ mutation (Arg166Pro) ${ }^{19}$ and three new mutations (Phe 237Ser, c.1442 dup A, and Glu460Asp) from the 135 cardiomyopathy patients studied. The main clinical characteristics associated with these mutations are shown in Table 1.

The Glu460Asp mutation was found in a large family with 13 mutation carriers, the pedigree of the family is shown in Figure 1. The mutation was predicted to be benign using PolyPhen-2 with a score of 0.174 (sensitivity: 0.93 ; specificity: 0.87 ). The phenotype varied from the proband's classical DCM with a left ventricular ejection fraction (LVEF) of $20 \%$ at the time of diagnosis to completely asymptomatic. The most common findings among the carriers of the mutation were atrioventricular blocks necessitating pacemaker implantation. Family-members not carrying the Glu460Asp mutation did not display any symptoms or signs or cardiomyopathy. Classifying familymembers with atrioventricular blocks, arrhyth-

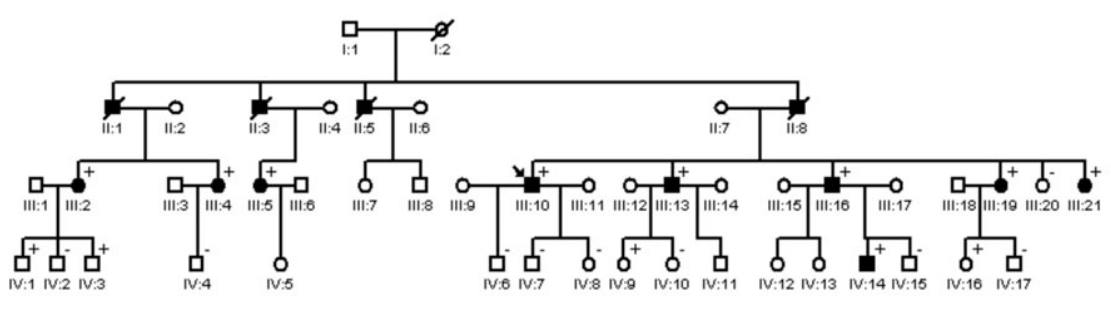

Figure 1. Pedigree of the family affected with the Glu460Asp LMNA mutation. Circles represent women; squares men, black-filled symbols represent clinically affected; plus sign, presence of the Glu460Asp LMNA mutation; minus sign, absence of the mutation.

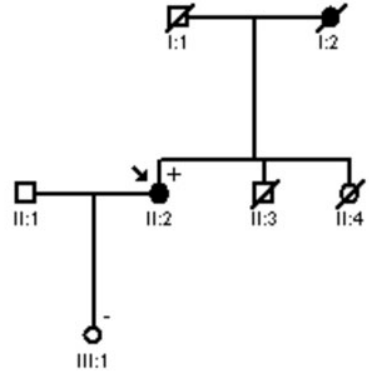

Figure 2. Pedigree of the family affected with the Arg166Pro $L M N A$ mutation. Circles represent women; squares men, black-filled symbols represent clinically affected; plus sign, presence of the Arg166Pro LMNA mutation; minus sign, absence of the mutation. 
mias or DCM as affected, and estimating linkage with Glu460Asp based on a parametric affected-only model, yielded a LOD [logarithm (base 10) of odds] score of 2.96. The Arg166Pro mutation (pedigree shown in Figure 2) was also associated with a typical cardiolaminopathy. PolyPhen-2 analysis predicted the mutation to be possibly damaging with a score of 0.950 (sensitivity: 0.79 ; specificity: 0.95$)$. In addition to DCM the proband suffers from progressive distal sensorymotor primary axonal polyneuropathy. The proband carrying a Lamin A/C mutation c.1442 dup A (pedigree shown in Figure 3) has never fully fulfilled the criteria for DCM; however, the course of her heart disease has been quite typical to laminopathy starting with atrial fibrillation at 41 and a non-sustained ventricular tachycardia and an implantable cardioverterdefibrillator implantation some 10 years later.

The fourth Lamin A/C mutation Phe237Ser (pedigree shown in Figure 4) is associated with an unusual cardiac phenotype affecting primarily the right chambers of the heart. The mutation is located in a highly conserved part of the linker 2 domain of the protein (Figure 5). PolyPhen-2 analysis predicted the mutation to be possibly damaging with a score of 0,691 (sensitivity: 0.86 ; specificity 0.92 ). We present six proven carriers and two obligatory carriers of the mutation. The mutation was not found in any of the 186 healthy Finnish controls.

Five family members had severe tricuspid insufficiency, right ventricular enlargement and right ventricular failure. Also the right atrium was enlarged in some of the affected patients. Moderate mitral valve regurgitation was present in three patients.

The proband (III:4) started showing symptoms of heart disease at the age of 43 . He had mild chest pain and dyspnea even at rest. Cardiac ultrasound showed initially a dilated, hypokinetic right ventricle, a dilated right atrium, a moderately dilated left ventricle, a severe tricuspid insufficiency, and an LVEF of $40 \%$. He had paroxysms of atrial fibrillation soon progressing to chronic atrial fibrillation. Coronary angiography showed no signs of coronary artery disease. The tricuspid insufficiency was first considered as the primary cause of right ventricular failure, and because he was symptomatic, a mechanical tricuspid valve was installed. The main finding in the valve operation was a dilated tricuspid valve annulus. Despite successful replacement of the tricuspid valve, the patient remained symptomatic. Two years after the first signs of heart disease echocardiography showed that the LVEF had diminished to $35 \%$. In follow-up the heart failure progressed. Coronary angiography showed still no signs of coronary artery disease (CAD). At the age of 47 he received a heart transplant. The histological examination of the explanted heart showed fibrotic replacement of the

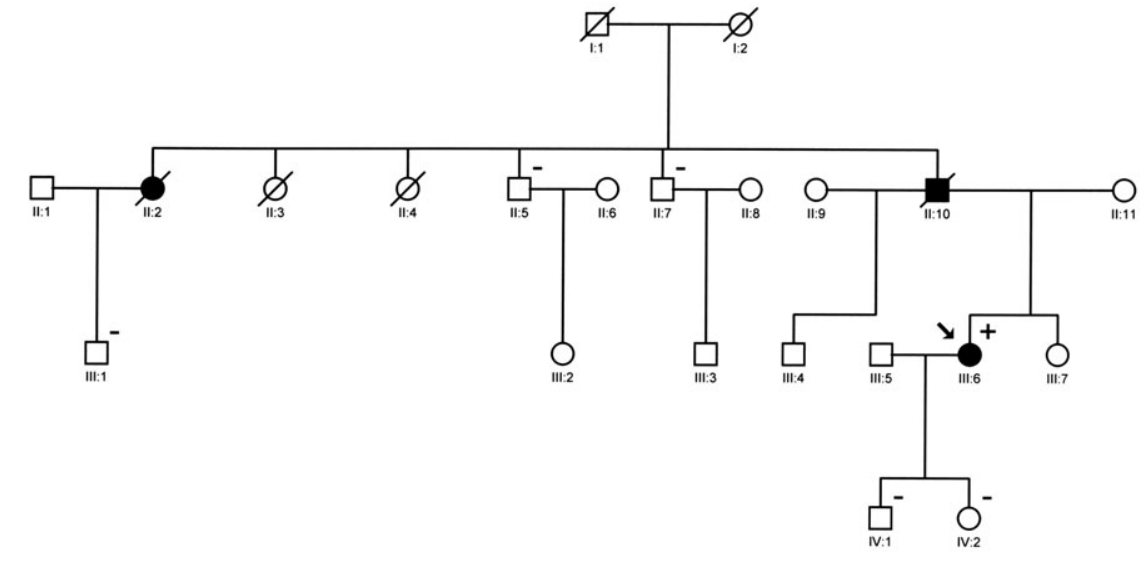

Figure 3. Pedigree of the family affected with the c.1442 dup A LMNA mutation. Circles represent women; squares men, black-filled symbols represent clinically affected; plus sign, presence of the c.1442 dup A LMNA mutation; minus sign, absence of the mutation.

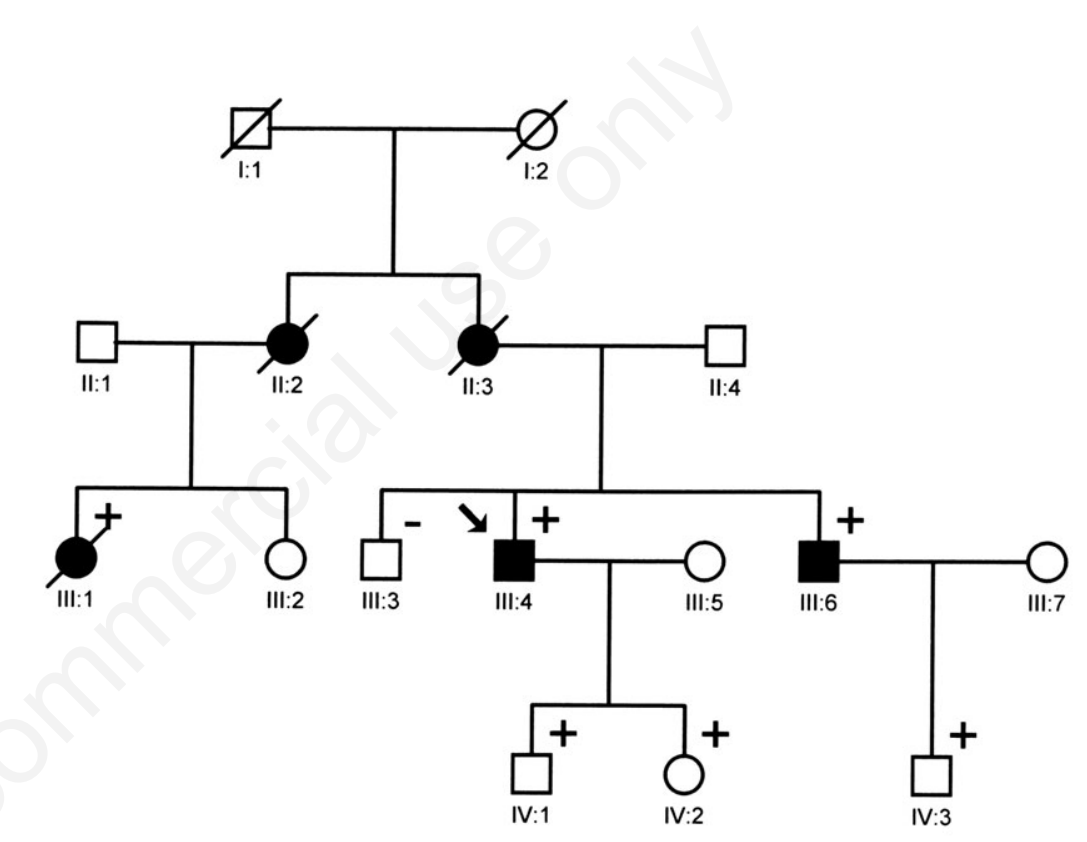

Figure 4. Pedigree of the family affected with the Phe237Ser LMNA mutation. Circles represent women; squares men, black-filled symbols represent clinically affected; plus sign, presence of the Phe237Ser LMNA mutation; minus sign, absence of the mutation.

\begin{tabular}{|c|c|c|c|c|c|c|c|c|c|c|c|}
\hline HUMAN & G & K & Q & R & E & F & E & S & R & L & A \\
\hline RHESUS & G & K & Q & R & E & F & E & S & R & L & A \\
\hline MOUSE & G & K & Q & R & E & F & E & S & R & L & A \\
\hline DOG & G & K & Q & R & E & F & E & S & R & L & A \\
\hline ELEPHANT & G & K & Q & R & E & F & E & S & R & L & A \\
\hline OPOSSUM & G & K & Q & R & E & F & E & N & K & L & A \\
\hline X_TROPICALIS & G & R & Q & R & E & F & E & S & K & L & A \\
\hline ZEBRAFISH & G & R & Q & Q & D & Y & E & S & K & L & A \\
\hline
\end{tabular}

Figure 5. Conservation of the mutated amino acid Phe237Ser whose location is denoted in red color. Sequences are aligned according to University of California, Santa Cruz (UCSC) Genome Browser Vertebrate Multiz Alignment \& Conservation. 
myocardium especially in the right atrium.

The proband's brother (III:6) had atrial fibrillation and signs of heart failure when he was 41 years old. Initially the echocardiogram showed hypokinesia, a mild mitral insufficiency and a significant tricuspid insufficiency. As his brother, he also had atrial fibrillation. At an attempted cardioversy, he went to asystole, and consequently received a pacemaker. Less than three years later the tricuspid insufficiency had progressed to a severe stage. At this point all the chambers of his heart were dilated. Angiography showed no signs of CAD. Shortly after his brother, he received a heart transplant at the age of 44 .

The proband's cousin (III:1) presented with a similar heart disease at 44 . She had right ventricular failure with right ventricular dilatation and chronic atrial fibrillation. Left ventricular diameter was normal. Electron microscopy of the endomyocardial biopsy showed unspecific degenerative changes in addition to nuclear blebbing, which is commonly seen in Lamin $\mathrm{A} / \mathrm{C}$ mutated cells, but not in normal cells ${ }^{24}$ (Figure 6). Eighteen months after the initial symptoms the patient received a pacemaker because of a symptomatic bradycardia. At date, electrocardiogram showed virtually no atrial activity. In the course of some three years the right ventricular failure progressed. However, due to follicular lymphoma, she was not a candidate for a heart transplant. Two years later, she died of heart failure. Six months prior to her death she suffered a mild cardiogenic stroke.

According to hospital records the proband's mother (II:3), an obligatory mutation carrier, had a high blood pressure before she was 30 . At 43 she received a pacemaker due to atrial fibrillation and sick sinus syndrome. Six months later she had a clinically diagnosed transient ischemic attack. At 48 she suffered a subarachnoidal haemorrhage and underwent surgery of the ruptured aneurysm. At 53 she was diagnosed with clinical heart failure. Initially the most important echocardiographical finding was a significantly dilated right side of the heart and a tricuspid insufficiency. In addition she had a mild combined aortic valve defect. Due to the failure of the right side of the heart she had liver cirrhosis. She also developed an end-stage renal failure and died at 56. Autopsy was not performed.

The proband's aunt (II:2) received a pacemaker at 52 due to sick sinus syndrome and an atrioventricular block. Echocardiography showed initially a combined aortic valve defect, mild mitral insufficiency and a massive tricuspid insufficiency. At 58 she underwent mitral valvuloplasty and tricuspid valvuloplasty. However surgery did not alter the outcome of her heart disease, and 4 months after surgery she died of heart failure.

The proband's 24-year-old son (IV:1), 28-

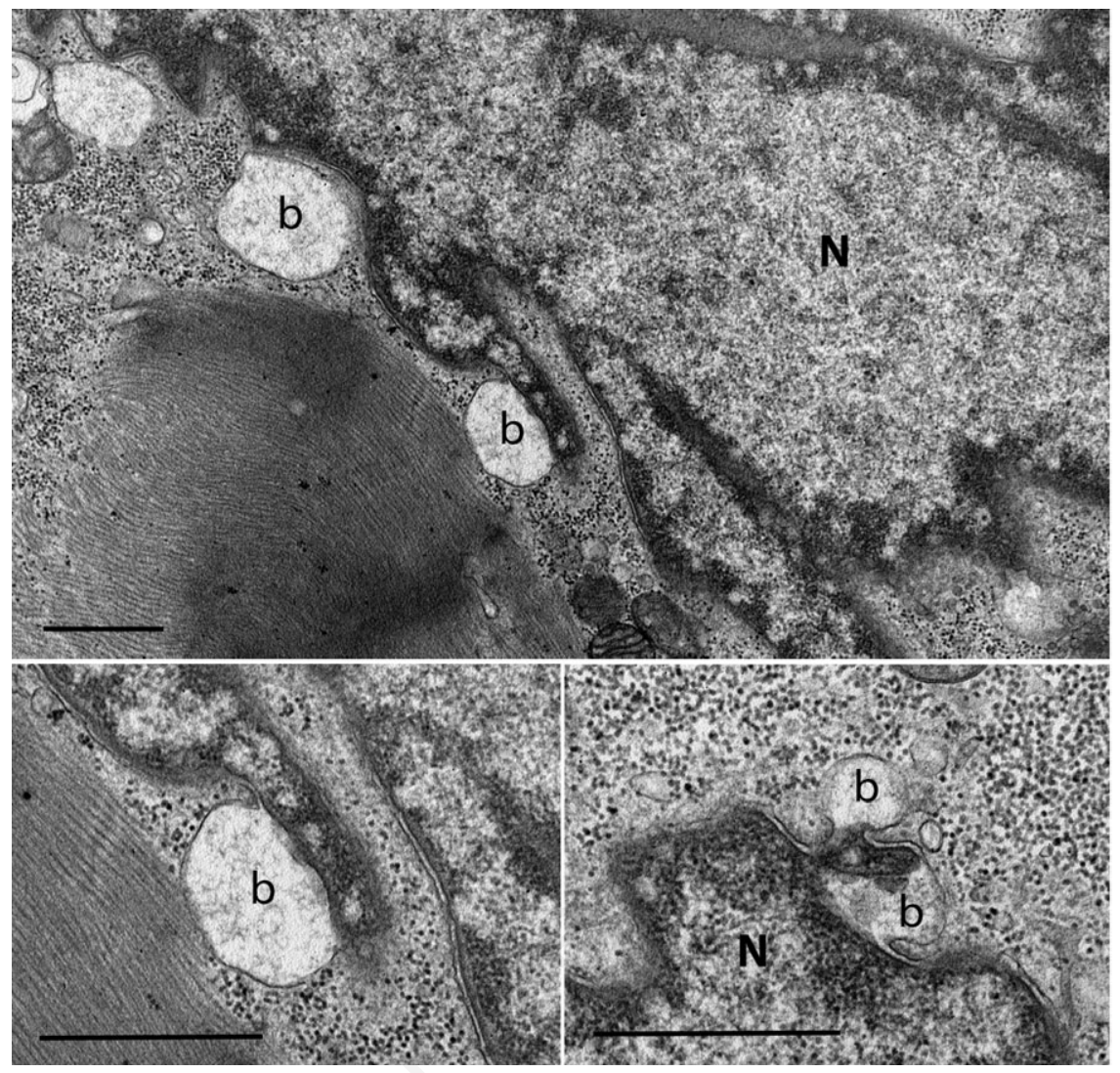

Figure 6. Electron microscopy of endomyocardial biopsy (of patient III:1 of the family affected with the Phe237Ser LMNA mutation). The shape of the cardiomyocyte nucleus $(\mathrm{N})$ in the upper panel is extensively convoluted and the nuclear membrane shows herniations or blebs (b) to the cytoplasm. The lower left panel is a detail from the upper panel. The lower right panel shows another example of nuclear blebs in a cardiomyocyte nucleus. The scale bar corresponds to $1 \mu \mathrm{m}$.
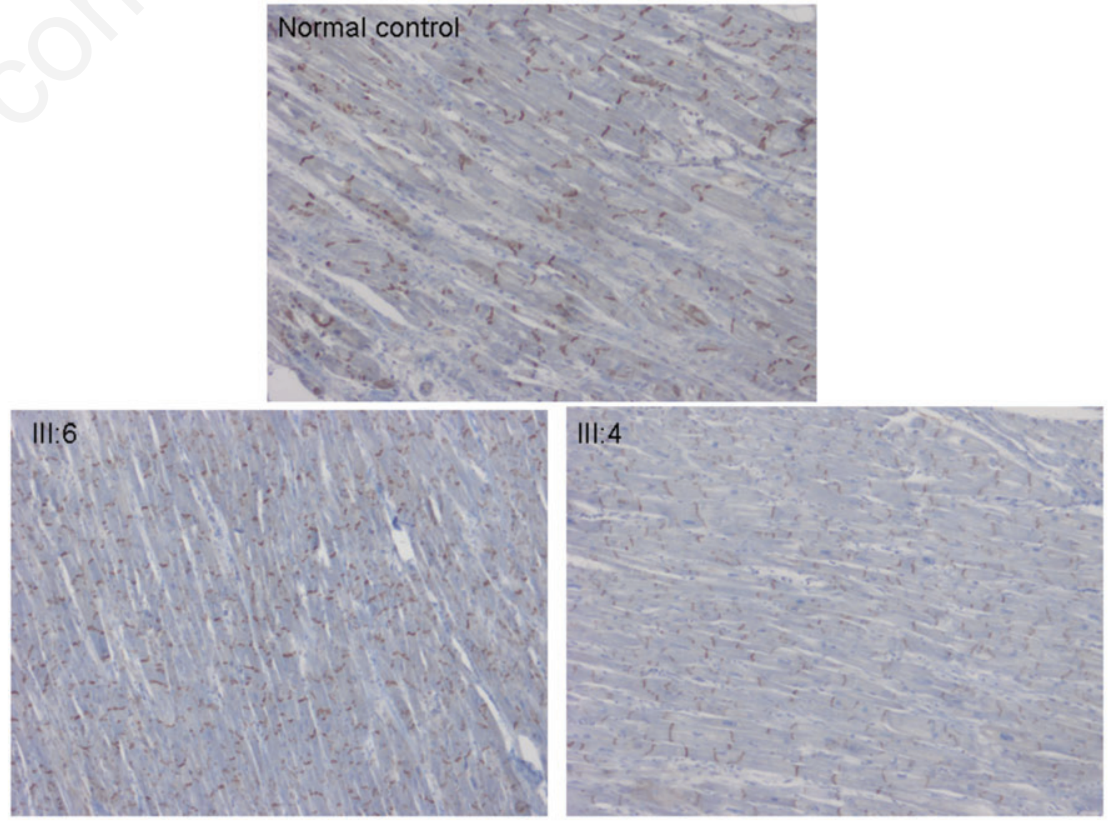

Figure 7. Immunohistochemical staining for the desmosomal protein plakoglobin in heart muscle biopsies (of patients III:4 and III:6 of the family affected with the Phe237Ser $L M N A$ mutation). Intercalated discs show normal signal levels in a normal control sample and in both patient samples. 
year-old daughter (IV:2), and 22-year-old nephew (IV:3) are all carriers of the mutation Phe237Ser. To date, they have shown no symptoms of heart disease and have normal echocardiographic findings as well as normal findings in cardiac magnetic resonance imaging. The only cardiac abnormality found in the proband's 22-year-old nephew is an unspecific intraventricular conduction abnormality and a slightly reduced performance in cardiac stress test. The proband has a second brother (III:3) who has no symptoms of heart disease. He turned out to be the only family member who is not a carrier of the new Lamin $\mathrm{A} / \mathrm{C}$ mutation.

The plakoglobin staining pattern of the

Table 1. The clinical manifestations associated with the LMNA mutations Glu460Asp, Arg166Pro, c. 1442 dup A and Phe237Ser. Probands are marked in italics.

\begin{tabular}{|c|c|c|c|c|c|c|c|c|c|}
\hline $\begin{array}{l}\text { Mutation } \\
\text { mutation } \\
\text { carrier }\end{array}$ & $\begin{array}{l}\mathrm{F} / \mathrm{M} \text {, } \\
\text { age }\end{array}$ & $\begin{array}{c}\text { DCM/ } \\
\text { HF }\end{array}$ & $\begin{array}{l}\text { LVEF } \\
\text { lowest }\end{array}$ & $\begin{array}{l}\text { LVEDD } \\
\max \end{array}$ & $\begin{array}{c}\text { Right } \\
\text { heart } \\
\text { involvement } \\
\text { (atrium, } \\
\text { ventricle, } \\
\text { valves) }\end{array}$ & Transplant & ECG & PM & Other \\
\hline \multicolumn{10}{|c|}{ Glu460Asp, pedigree shown in Figure 1} \\
\hline III:2 & $\mathrm{F} / 61$ & n. & 51 & 49 & No & No & SSS, DAV III & PM & \\
\hline III:4 & $\mathrm{F} / 53$ & n. & 61 & 58 & No & No & DAV I & No & \\
\hline III:5 & $\mathrm{F} / 44$ & n. & 69 & 52 & No & No & Atrial and ventricular extrasystoles, DAV I & No & \\
\hline III: 10 & $\mathrm{M} / 61$ & DCM & 20 & 59 & No & No & FA, bradycardia, DAV III & PM & \\
\hline III:13 & $\mathrm{M} / 60$ & No & 55 & 58 & No & No & FA, bradycardia, DAV III & PM & \\
\hline III:16 & $\mathrm{M} / 64$ & No & 61 & 56 & No & No & FA, bradycardia, DAV III & PM & \\
\hline III:19 & $\mathrm{F} / 54$ & No & 58 & 56 & No & No & FA, focal atrial tachycardia & No & \\
\hline III:21 & $\mathrm{F} / 56$ & No & 53 & 52 & No & No SSS & S, FA, atrial extrasystoles, bradycardia, DAV III & PM & \\
\hline IV:1 & $\mathrm{M} / 32$ & No & 62 & 51 & No & No & 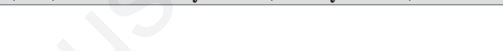 & No & \\
\hline IV:3 & $\mathrm{M} / 33$ & No & 58 & 51 & No & No & 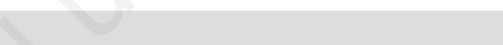 & No & \\
\hline IV:9 & $\mathrm{F} / 34$ & No & 63 & 55 & No & No & Unconducted p waves in Holter & No & \\
\hline IV:14 & $\mathrm{M} / 23$ & No & 53 & 54 & No & No & DAV I & No & \\
\hline IV:16 & $\mathrm{F} / 17$ & No & 70 & 52 & No & No & & No & \\
\hline \multicolumn{10}{|c|}{ Arg166Pro, pedigree shown in Figure 2} \\
\hline $\mathrm{I}: 2$ & F/deceased & & & & & No & SVT, DAV III & PM & \\
\hline$I I: 2$ & $\mathrm{~F} / 71$ & DCM & 25 & 54 & No & Yes & FA, DAV III & PM & $\begin{array}{c}\text { Progressive } \\
\text { distal } \\
\text { sensorymotor } \\
\text { primary axonal } \\
\text { polyneuropathy }\end{array}$ \\
\hline
\end{tabular}

\section{c.1442 dup A, pedigree shown in Figure 3}

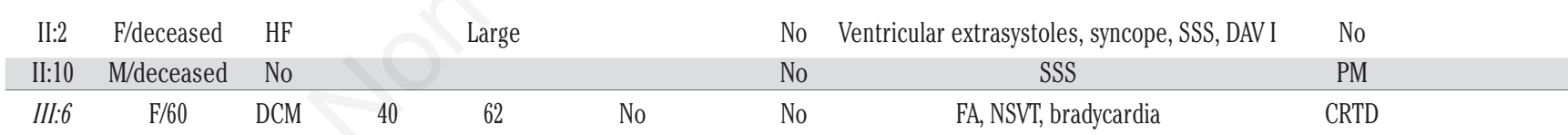

\section{Phe237Ser, pedigree shown in Figure 4}

\section{II:2 F/deceased HF}

\begin{tabular}{ccccc} 
II:3 & F/deceased & HF & 56 & 43 \\
III:1 & F/deceased & HF & & \\
III:4 & M/54 & HF & 30 & 58 \\
& & & & \\
\hline III:6 & M/51 & HF & 20 & 62 \\
IV:1 & M/24 & No & 67 & 50 \\
IV:2 & $\mathrm{F} / 28$ & No & $>50$ & 43 \\
IV:3 & M/22 & No & 50 & 52
\end{tabular}

Dilated RV, RA, TI, MI. $\quad$ No Tricuspid and mitral plasties

\section{Dilated RA, RV,}

$$
\text { TI, RV failure No }
$$

RV dilatation and failure No

8 Dilated RV, RA, severe TI, mechanic tricuspid valve

Severe TI

$$
\text { Yes }
$$

FA, SSS

SSS, DAV grade nd

PM

, male; F, female; DCM, dilated cardiomyopathy; HF, heart failure; LVEF, left ventricular ejection fraction; LVEDD, left ventricular end diastolic diameter; ECG, electrocardiogram; PM, pacemaker; SSS, sick sinus syndrome; DAV, atrioventricular block; FA, atrial fibrillation; SVT, supraventricular tachycardia; NSVT, non-sustained ventricular tachycardia; CRTD, cardiac resynchronization therapy defibrillator; SCD, sudden cardiac death; TI, tricuspid insufficiency; RA, right atrium; RV, right ventricle; TI, tricuspid insufficiency; MI, mitral insufficiency; EMB, endomyocardial biopsy. 
myocardial samples of the proband (III:4) and his brother (III:6) was normal therefore suggesting no underlying desmosomal pathology. (Figure 7) In addition, the proband's brother (III:6) and his cousin (III:1) had no PKP2 mutations. Nor did they have any of the previously identified Finnish desmosomal mutations (PKP2 Q59L, PKP2 Q62K, PKP2 N613K, DSP T1373A and DSG2 3059_3062del AGAG). ${ }^{18,22}$

\section{Discussion and Conclusions}

We describe one previously reported and three new lamin $\mathrm{A} / \mathrm{C}$ mutations. The Glu460Asp mutation was found in a family with 9 affected mutation carriers. The oldest of the four unaffected mutation-carriers is 33 years old, which is in accordance with the agedependent penetrance of cardiolaminopathies. Even if the mutation showed complete co-segregation with atrioventricular blocks, arrhythmias or DCM, the obtained LOD score, 2.96, is just short of statistical significance due to the limited number of affected meioses available in the pedigree. In regard to the mutations Arg166Pro and c. 1442 dup A, the families were small and only the probands were shown to be mutation carriers. However, as reported in Table 1, both probands had a familial cardiomyopathy with a phenotype quite typical to cardiolaminopathy. Polyphen analysis predicted the Arg166Pro mutation to be possibly damaging. These findings support the idea that the mutations are pathogenic. Our main finding was a new Phe237Ser mutation of Lamin $\mathrm{A} / \mathrm{C}$ gene in patients with a severe cardiomyopathy affecting primarily the right side of the heart. The mutation resides in a conserved location of the LMNA gene, and appears to be disease-causing as it co-segregates with the phenotype and was not present in healthy controls. The proband, his brother and their female cousin all had severe cardiac disease by their early forties, whereas the proband's other brother, who was not a mutation carrier, showed no signs of cardiac disease. The endomyocardial biopsy of the proband's cousin showed nuclear blebbing, which is a typical finding in cardiolaminopathy. The other three proven carriers who are still healthy are all under 30 , which is in accordance with the age-dependent penetrance of cardiolaminopathies. ${ }^{9,10}$

The Phe237Ser LMNA mutation carriers presented with a phenotype affecting the right side of the heart, but not fulfilling the current Task Force criteria for ARVC. ${ }^{25}$ In addition, the pathological changes, i.e. dilatation of the right atrium and ventricle as well as tricuspid valve insufficiency, were not typical for ARVC. To further exclude ARVC, we screened the proband's cousin and brother for the Finnish
ARVC mutations, which were not found. However, as a definitive exclusion of ARVC by DNA diagnostics is not possible, and since a reduction in immunoreactive signal levels for plakoglobin has been introduced as a possible diagnostic tool for ARVC, ${ }^{26,27}$ we performed immunohistochemical analysis for the myocardial samples of the proband and his brother. The immunohistochemical staining pattern was normal. Thus, our observations suggest that the phenotype described here is not caused by malfunctioning desmosomes. Our finding differs from those of Quarta et al. who recently reported abnormal plakoglobin staining of intercalated discs in an individual with ARVC due to LMNA mutation Gly382Val. ${ }^{17}$

In conclusion, the Phe237Ser mutation in Lamin A/C causes a severe phenotype of cardiolaminopathy leading to early death or heart transplantation. This finding suggests that Lamin A/C mutations should be considered when a patient presents with a cardiomyopathy affecting primarily the right side of the heart. This is in line with the recommendation Quarta et al. made in their recent work reporting LMNA mutations in ARVC patients. ${ }^{17}$ In addition, our findings reflect the clinical challenge of dividing cardiomyopathies to their traditional categories and demonstrate the overlapping genetic background of cardiomyopathy phenotypes.

\section{References}

1. Elliott P, Andersson B, Arbustini E, et al. Classification of the cardiomyopathies: A position statement from the European society of cardiology working group on myocardial and pericardial diseases. Eur Heart J 2008;29:270-6.

2. Burkett EL, Hershberger RE. Clinical and genetic issues in familial dilated cardiomyopathy. J Am Coll Cardiol 2005;45: 969-81.

3. Dellefave L, McNally EM. The genetics of dilated cardiomyopathy. Curr Opin Cardiol 2010;25:198-204.

4. Hershberger RE, Morales A, Siegfried JD. Clinical and genetic issues in dilated cardiomyopathy: A review for genetics professionals. Genet Med 2010;12:655-67.

5. Flack E, Kannankeril PJ. The genetics of dilated cardiomyopathy. Heart Rhythm 2012;9:397-8.

6. Herman DS, Lam L, Taylor MRG, et al. Truncations of titin causing dilated cardiomyopathy. NEJM 2012;366:619-28.

7. Kärkkäinen S, Reissel E, Heliö T, et al. Novel mutations in the lamin $\mathrm{A} / \mathrm{C}$ gene in heart transplant recipients with end-stage dilated cardiomyopathy. Heart 2006;92: 524-6.
8. Kärkkäinen S, Heliö T, Miettinen R, et al. A novel mutation, Ser143Pro, in the lamin $\mathrm{A} / \mathrm{C}$ gene is common in Finnish patients with familial dilated cardiomyopathy. Eur Heart J 2004;25:885-93.

9. van Berlo JH, de Voogt WG, van der Kooi $\mathrm{AJ}$, et al. Meta-analysis of clinical characteristics of 299 carriers of LMNA gene mutations: do lamin $\mathrm{A} / \mathrm{C}$ mutations portend a high risk of sudden death? J Mol Med 2005;83:79-83.

10. Pasotti M, Klersy C, Pilotto A, et al. Longterm outcome and risk stratification in dilated cardiolaminopathies. J Am Coll Cardiol 2010;52:1250-60.

11. Jacoby D, McKenna WJ. Genetics of inherited cardiomyopathy. Eur Heart J 2012;33:296-304.

12. Elliott P, O'Mahony C, Syrris P, et al. Prevalence of desmosomal protein gene mutations in patients with dilated cardiomyopathy. Circ Cardiovasc Genet 2010;3:314-22.

13. Herren T, Gerber PA, Duru F. Arrhythmogenic right ventricular cardiomyopathy/dysplasia: a not so rare "disease of the desmosome" with multiple clinical presentations. Clin Res Cardiol 2009;98:141-58.

14. Tiso N, Stephan DA, Nava A, et al. Identification of mutations in the cardiac ryanodine receptor gene in families affected with arrhythmogenic right ventricular cardiomyopathy type 2 (ARVD2). Hum Mol Genet 2001;10:189-94.

15. Beffagna G, Occhi G, Nava A, et al. Regulatory mutations in transforming growth factor-beta3 gene cause arrhythmogenic right ventricular cardiomyopathy type 1. Cardiovasc Res 2005;65:366-73.

16. Merner ND, Hodgkinson KA, Haywood AF, et al. Arrhythmogenic right ventricular cardiomyopathy type 5 is a fully penetrant, lethal arrhythmic disorder caused by a missense mutation in the TMEM43 gene. Am J Hum Genet 2008;82:809-21.

17. Quarta G, Syrris P, Ashworth M, et al. Mutations in the Lamin $\mathrm{A} / \mathrm{C}$ gene mimic arrhythmogenic right ventricular cardiomyopathy. Eur Heart J 2012;33:1128-36.

18. Lahtinen AM, Lehtonen E, Marjamaa A, et al. Population-prevalent desmosomal mutations predisposing to arrhythmogenic right ventricular cardiomyopathy. Heart Rhythm 2011;8:1214-21.

19. Saga A, Karibe A, Otomo J, et al. Lamin A/C gene mutations in familial cardiomyopathy with advanced atrioventricular block and arrhytmia. Tohoku J Exp Med 2009;218:309-16.

20. Van Tintelen JP, Hofstra RM, Katerberg H, et al. High yield of LMNA mutations in patients with dilated cardiomyopathy and/or conduction disease referred to car- 
diogenetics outpatient clinics. Am Heart J 2007;154:1130-9.

21. Adzhubei IA, Schmit S, Peshkin, et al. A method and server for predicting damaging missense mutations. Nat Methods 2010;7:248-9.

22. Lahtinen AM, Lehtonen A, Kaartinen M, et al. Plakophilin-2 missense mutations in arrhytmogenic right ventricular cardiomyopathy. Int J Cardiol 2008;126:92-100.

23. Abecasis GR, Cherny SS, Cookson WO,
Cardon LR. Merlin-rapid analysis of dense genetic maps using sparse gene flow trees. Nat Genet 2002;30:97-101.

24. Smith ED, Kudlow BA, Frock RL, et al. Atype nuclear lamins, progerias and other degenerative disorders. Mech Aging Dev 2005;126:447-60.

25. Marcus FI, McKenna WJ, Sherrill D, et al. Diagnosis of arrhythmogenic right ventricular cardiomyopathy/dysplasia: proposed modification of the task force crite- ria. Circulation 2010;121:1533-41.

26. Asimaki A, Tandri H, Huang $\mathrm{H}$, et al. A new diagnostic test for arrhythmogenic right ventricular cardiomyopathy. NEJM 2009;360:1075-84.

27. Munkholm J, Christensen AH, Svendsen $\mathrm{JH}$, Andersen CB. Usefulness of immunostaining for plakoglobin as a diagnostic marker of arrhythmogenic right ventricular cardiomyopathy. Am J Cardiol 2012;109: 272-5. 